\title{
Phytotherapeutic antioxidants
}

\author{
Tulika Mishra ${ }^{1}$, Ananda Kumar Kondepati², Shalini Devaprasad Pasumarthi ${ }^{3}$, \\ Gurmit Singh Chilana ${ }^{4}$, Suresh Devabhaktuni ${ }^{5}$, Pratik Kumar Singh ${ }^{6}$
}

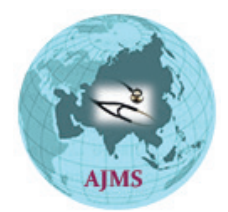

${ }^{1}$ Assistant Professor, ${ }^{3}$ Professor, Department of Microbiology, ${ }^{2}$ Professor and Director, Department of Physiology, ${ }^{4}$ Chief Academic Officer, Department of Obstretics and Gynecology, ${ }^{5}$ Assistant Professor, Department of Pharmacology,

${ }^{6}$ MBBS Student, Aureus University School of Medicine, Oranjestad, Aruba, Dutch Caribbean

Plants have always been an integrated part of human life, whether we discuss about providing food or about their medicinal value. Ethnomedicines have led to the discovery of many valuable drugs against various diseases. There are many diseases that are affected by imbalance balance between antioxidants and prooxidants or free radicals. Free radicals are the chemical entities capable of independent existence that contain one/more unpaired electrons and can be balanced by antioxidants. Antioxidants maintain the integrity and function of membrane lipids, cellular proteins, and nucleic acids and the control of signal transduction of many genes. Whenever there is imbalance between prooxidant and antioxidants they can lead to many diseases and even ameliorate the severity of disease like Alzheimer, cardiovascular disorder, cancer etc. There are evidences showing usage of plants as a potent source of antioxidants has provided a lot of support in combating various diseases, without any side effect. Various Bioflavonoids, Carotenoids, Hydroxycinnamates etc helps in scavenging these free radicals. There are various mechanisms through which these Phyto antioxidants assisted against various diseases. The present review is an attempt showing the relationship of free radicals with various diseases and the role of Phyto antioxidants against various health related conditions.

Key Words: Antioxidants; Plants; Free radicals; Diseases

\section{Access this article online}

Website:

http://nepjol.info/index.php/AJMS DOI: 10.3126/ajms.v11i2.26465 E-ISSN: 2091-0576 P-ISSN: $2467-9100$

\section{INTRODUCTION}

Plants have played a significant role in maintaining human health and improving the quality of life since thousands of years. There is an ever-growing interest in investigating different species of plants to identify their potential therapeutic applications due to a tremendous historical legacy in folkloric use of plants as medicine and their easy availability, cost effectiveness, presumed safety and biological friendliness than totally synthetic molecules. ${ }^{1}$ Ayurveda remains one of the most ancient and yet living traditions practiced widely in India, Srilanka and other countries and has a sound philosophical and experimental basis. Atharvaveda (around 1200 BC), Charak Sambita and Sushrut Sambita (1000-500 BC) are the classics that give detailed descriptions of over 1000 herbs. $^{2}$

In the recent past, the scientific studies on the plants used in ethnomedicine have led to the discovery of many valuable drugs such as pilocarpine, vincristine, etoposide and paclitaxel. With the advancement in technology and move towards a progressive life, humankind is heading towards many diseases like cancer, AIDS, tuberculosis, diabetes, chronic inflammatory diseases, atherosclerosis and neurodegenerative diseases. One of the major causes of many of these diseases is oxidative stress caused by imbalance between antioxidants and prooxidants. Prooxidants or free radicals are the species capable of independent existence that contain one/more unpaired electrons. It is well known that oxygen plays an important role in many metabolic processes associated with aerobic existence while on the other hand, it leads to the formation of reactive oxygen species that have either unpaired electrons (e.g., $\mathrm{O}_{2}{ }_{2}, \mathrm{OH}^{*}$ ) or the ability to attract electron from various molecules like $\mathrm{H}_{2} \mathrm{O}_{2}$. Free radicals not only contribute to the elimination of infected cells, but when present in higher concentrations react with cellular 
DNA, proteins, lipids and other macromolecules. The disease such as brain dysfunction, cancer, autoimmune pathologies, gastrointestinal inflammation and ulcers, ${ }^{3}$ heart diseases and immune decline could be due to these free radicals. ${ }^{4,5}$ In physiological condition, the human body can compensate for a mild degree of oxidant stress and remove oxidatively damaged molecules by activating antioxidant enzymes like superoxide dismutase, catalase, glutathione peroxidase, etc. Thus, owing to their ability to reduce oxidative damage by delaying or inhibiting lipid peroxidation or oxidation chain reaction, antioxidants have been of interest of pharmacologists, biochemists and other health professionals. Nowadays, there is an increasing interest towards replacing synthetic antioxidants with herbal remedies due to possible carcinogenic and immune decline effects of synthetic antioxidants. ${ }^{6}$ The major drawbacks of most of the chemotherapeutic agents available today are cytotoxic and exert variety of side effects. In contrast, herbal drugs or Phytotherapeutic agents have been found safer, and combating side effects is the paramount of treatment. Observing the beneficial role of Phyto antioxidants the present review is an attempt where various immune related disease, their relationship with free radicals and Phyto antioxidants has been focused.

\section{FREE RADICALS AND PHYTOANTIOXIDANTS}

Free Radicals are the chemical entitites capable of independent existence that contain one ore unpaired electrons. Free radicals attack all the major classes of molecules and mainly polyunsaturated fatty acids (PUFA), and this process is termed as lipid peroxidation (LPO). Lipid peroxidation is a destructive self-perpetuating chain reaction. ${ }^{7}$ In the PUFA free radicals first targets reactive lipid hydrogen $(\mathrm{LH})$ and oxidizes $\mathrm{LH}$ to fatty acid radical $(\mathrm{L})$, this fatty acid radical adds oxygen to form a fatty acid peroxyl radical (LOO). The peroxy radicals than induces further chain reaction. This peroxy radical further oxidize PUFA molecule and initiates new chain reaction producing lipid hydroperoxide ( $\mathrm{LOOH}$ ) which will further break down to more radical and reactive aldehyde, which further spread the attack to other parts of the cell. ${ }^{8}$

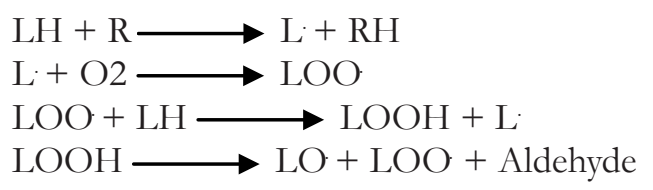

Lipid peroxidation is widely associated with injuries and diseases.

In addition to other free radicals, Reactive oxygen species (ROS) is most commonly produced in our metabolic system which includes all highly reactive, oxygen-containing molecules. Types of ROS include the hydroxyl radical, the super oxide anion radical, hydrogen peroxide, singlet oxygen, nitric oxide radical, hypochlorite radical, and various lipid peroxides. They are neutral, short lived, unstable and highly reactive to pair up the odd electron and finally achieve stable configuration. They are capable of attacking the healthy cells of the body, causing them to lose their structure and function. There are various endogenous (Metabolic process) and Exogenous (Environmental) sources of free radicals. Ccellular damage arising from reactive oxygen species (ROS), at least in part, is responsible for the etiology and pathophysiology of various neurodegenerative disorders (e.g., Alzheimer disease, Parkinson disease, multiple sclerosis, Down's syndrome) and other diseases like inflammation, viral infections, autoimmune pathologies, and digestive system disorders such as gastrointestinal inflammation and ulcer. ${ }^{6,9}$ In living systems, free radicals are generated as part of the body's normal metabolic process in the mitochondrial respiratory chain, liver mixed function oxidases, by bacterial leucocytes, through xanthine oxidase activity, atmospheric pollutants, and from transitional metal catalysts, drugs and xenobiotics. In addition, chemical mobilization of fat stores under various conditions such as lactation, exercise etc. can result in increased radical activity and damage, in particular, to the immune and nervous systems. The stress hormones (adrenalin and noradrenalin) secreted by the adrenal glands too, may be metabolized into simpler, albeit, free radicals. In carcinogenesis, reactive oxygen species are responsible for initiating the multistage carcinogenesis process starting with DNA damage and accumulation of genetic events in one or few cell lines which leads to progressively dysplastic cellular appearance, deregulated cell growth, and finally carcinoma. ${ }^{10,11}$

To protect the cells and organ systems of the body against ROS, humans have evolved a highly sophisticated and complex antioxidant protection system, that functions interactively and synergistically to neutralize free radicals. Under natural conditions a dynamic balance between the free radicals and antioxidants is maintained. The amount of antioxidant principles present under normal physiological conditions may be insufficient to neutralize extra burden of free radicals. Therefore, antioxidants can be given from external sources like food etc., to protect against harmful diseases and to prevent, delay or ameliorate many of these disorders. ${ }^{12}$ Hence there has been an increased interest in the food industry and in preventive medicine in the development of "Natural antioxidants" as alternative sources. Plant materials being most acceptable by consumer especially have gained an interest as antioxidant supplements in the foods and drugs. Phytochemicals with antioxidant activity subsist extensively in fruits, vegetables, cereal grains, edible macro fungi, microalgae, and medicinal plants. ${ }^{13,14}$ Antioxidants are molecules that have the capacity 
to either inhibit or quench the free radicals and induce delay or inhibit cellular damage. This defense mechanism is an intricate part of the survival in all the species. These antioxidants can be categorized as enzymatic and nonenzymatic form or water soluble and lipid soluble or small molecule or large molecule. The enzymatic antioxidants break down $\&$ remove free radicals. Thus, convert oxidative products to hydrogen peroxide and then water in the presence of cofactors. The nonenzymatic antioxidants like vitamin $\mathrm{C}$, plant polyphenols etc interrupt the free radicals chain reaction. The antioxidants that are derived from plants are called as Phyto antioxidants. The usage of Plants or plant derived components to prevent and treat diseases or to promote healthy life is termed as Phyto therapy. ${ }^{15}$ Phytochemicals are secondary bioactive non-nutrient plant chemicals found in fruits, vegetables, and other plant foods that have health-related effects. Up to now, about 200,000 phytochemicals have been identified and 20,000 of them are derived from fruits, vegetables and grains. ${ }^{16} \mathrm{To}$ combat or reduce the harmful effect of free radicals, Phyto antioxidants follow one of the mechanisms: (1) scavenging species that initiate peroxidation, (2) chelating metal ions such that they are unable to generate reactive species or decompose lipid peroxides, (3) quenching ${ }^{\circ} \mathrm{O}_{2}$ - preventing formation of peroxides, (4) breaking the autoxidative chain reaction, and/or (5) reducing localized $\mathrm{O}_{2}$ concentrations. ${ }^{17}$. To understand the mechanism of action of these Phyto antioxidants it is indispensable to comprehend the generation and damaging reactions of free radicals.

Among the endless list of the Phyto antioxidants, Vitamin E, Vitamin C, various Biofavonids, carotenoids, Allicin, Piperine and curcumin are commonly studied for their mechanism. Their mechanism mostly included the inhibition of lipid peroxidation and free radical scavenging.

\section{ROLE OF ANTIOXIDANTS AS THERAPEUTIC AGENTS}

Chronic diseases like cardiovascular diseases (CVD), diabetes, Obesity, Alzheimer's etc has become the global health problems, leading to death and disability to millions of people. So far, research has shown that toting up of fruits and vegetables to the patient's diet resulted in a very beneficial outcome. Overproduction of oxidants (reactive oxygen species and reactive nitrogen species) in the human body is involved in the pathogenesis of many chronic diseases. This imbalance can be handled by the antioxidants and since plants are natural source with almost no side effects, so provides better outcome against various chronic diseases. Fruits and vegetables consumption with high contents of antioxidant phytochemicals is proven to increase the antioxidant capacity of serum/plasma.

\section{PROTECTIVE ACTION ON CARDIOVASCULAR DISEASES}

In the developed countries CVD is one of the leading causes of death..$^{18}$ Overproduction of oxidants ameliorates the severity of the disease. Oxidative damage results into endothelial cell injuries and deleterious vasodilator effects. The polyphenols help in the improvement of endothelial function. ${ }^{19}$ Another study indicated that total flavonoids of Flos chrysanthemi showed vasodilating effects and protected vasodilator reactivity from oxidative stress, which was mediated by endothelium-derived hyperpolarizing factor. ${ }^{20}$ Macrophage scavenger receptors take up oxidized low-density lipoprotein to promote cholesterol ester accumulation and foam cell formation, which is another factor contributing to atherosclerotic disease. In animal models, quercetin attenuated atherosclerosis by interfering with foam cell formation and pro-oxidant/proinflammatory response, which were key proatherogenic activities of macrophages. ${ }^{21}$ In addition to this, polyphenols have also been reported to act in a multiple ways. It not only protects from oxidative stress but also involved in blood pressure reduction and inflammation decreasing action. In CVD, accumulation of vascular smooth muscle cells plays a key role in the formation and development of lesions in atherosclerosis, which has been reported to be relieved by the use of Dehydroglyasperin C. Another complication of CVD can be Platelet aggregation and adhesion under pathophysiological leading to thrombosis and blockage of coronary arteries. Polyphenols derived from blueberries has been reported to modify this molecular event. ${ }^{19}$ Other Phyto antioxidants like allicin, lycopene and crocin has been reported to inhibit platelet aggregation and protected oxidative stress-induced apoptosis of platelets. ${ }^{23}$ Allicin, an antioxidant organosulfur compound from garlic, was found to protect the cardiovascular system by inducing vasorelaxation and alleviating cardiac hypertrophy, angiogenesis, platelet aggregation, hyperlipidemia and hyperglycemia. ${ }^{24}$ Therefore, antioxidant phytochemicals can be a good candidate for preventing and decline down the CVD.

\section{PROTECTIVE ACTION AGAINST DIABETES}

Diabetes is another major health problem, characterized by chronic hyperglycemia, which further can lead to many microvascular and macrovascular complications. Due to hyperglycemia and hyperlipidemia diabetes is usually accompanied by ameliorated production of free radicals. ${ }^{25}$ In the study, it was found that usage of plant antioxidants including $\alpha$ - and $\gamma$-tocopherol, $\alpha$ - and $\beta$-carotene, lycopene, $\beta$-cryptoxanthin, lutein, zeaxanthin, retinol and ascorbic acid abetted to decrease the complications associated 
with diabetes. ${ }^{26}$ Additionally, the juices from raphanus, ajwain, sowa and amaranthus leaves were potent source of biological antioxidants and possessed capacity to mitigate starch induced postprandial glycemic burden and reduced glucose induced postprandial glycemic excursion in rats. ${ }^{27}$ Besides this, Sub-clinical grade inflammation also plays a significant role in the expansion of obesity-related insulin resistance. Polyphenols originated from grape products abridged obesity-mediated chronic inflammation to prevent metabolic diseases. Polyphenols act by blocking proinflammatory cytokines or endotoxin-mediated kinases and transcription factors to exert its antidiabetic activity. ${ }^{28}$ Curcumin due to its strong anti-inflammatory and antioxidant activities is also considered suitable for the prevention and amelioration of diabetes. Resveratrol was found to influence the expression of genes relevant to the development of type- 2 diabetes, such as by inducing expression of several $\beta$-cell genes and insulin expression in pancreatic $\alpha$-cells. ${ }^{29}$ Phytochemicals could prevent diabetes through regulating $\alpha$-glucosidase and lipase activities, reducing postprandial glycemic level, anti-inflammatory activity, improving pancreatic function and synergistic action with hypoglycemic drugs. 30,31

\section{PROTECTIVE ACTION ON ALZHEIMER'S DISEASE AND INFLAMMATORY BOWEL DISEASE}

Alzheimer's is a deteriorating neurological disorder characterized by cognitive decline and memory loss. ${ }^{32}$ The brain is believed to be susceptible to oxidative stress as there is relatively higher concentration of oxygen free radicals without proportionate levels of antioxidative defenses. This oxidative stress has been reported to be involved in the pathogenesis of dementia or Alzheimer's. ${ }^{33}$ The person suffering from Alzheimer's shows remarkable reduction of acetylcholine (ACh) levels in the hippocampus and cortex of the brain resulting in memory loss. Studies reveled that usage of Phyto antioxidants like flavonoid helped in the reduction of oxidant stress. In addition, quercetin showed protective effect against neurotoxicity of amyloid $\beta$-peptide that usually found to be at higher level in Alzheimer's. ${ }^{34}$ In addition, curcumin, catechins and resveratrol showed neuroprotective ability in Alzheimer's. ${ }^{36,}$ ${ }^{37}$ Furthermore, polyphenolic compounds of walnuts reduced the oxidant and inflammatory load on brain cells, improved inter-neuronal signaling, increased neurogenesis, as well as enhanced sequestration of insoluble toxic protein aggregates. ${ }^{37}$ Therefore, phytochemicals could protect against $\mathrm{AD}$ by reduction of oxidant stress and acetylcholinesterase.

Inflammatory bowel disease (IBD) includes two complex diseases viz; ulcerative colitis (UC) and Crohn's disease and both the disease are associated with chronic inflammatory disorder regulated by immune response. Previous studies showed that free radicals declines the immune status of host. ${ }^{38,4}$ Thereby, theses oxidants enhance the deleterious effect of IBD. ${ }^{39}$ Polyphenols like picatechin, procyanidin $\mathrm{B} 2$, catechin, and procyanidin $\mathrm{B} 1$ possess strong anti-inflammatory property. ${ }^{40-42}$

\section{CONCLUSION}

From the data available it can be concluded that many medical conditions where oxidative stress is involved can be well treated with various plants. These medicinal properties of the plant owe to its phytoconstituents. Most important aspect of theses Phyto antioxidants lies in the fact that being a natural source they have less or even no side effects. Although more research is required in the field of Phyto antioxidants so that new formulations can be explored.

\section{REFERENCES}

1. Balunas $\mathrm{MJ}$ and Kinghorn AD. Drug discovery from medicinal plants. Life Sci 2005; 78:431-441.

2. Holt GA and Chandra A. Herbs in the modern health care environment- An overview of uses, legalities and the role of the health care professional. Clin Res Regulatory Affairs (USA) 2002; 19:83-107.

3. Mishra T, Khullar $\mathrm{M}$ and Bhatia A. Antiscancer potential of aqueous ethanol seed extract of Ziziphus mauritiana against cancer cell lines and Ehrlich ascites carcinoma. Evid Based Complement Alternat Med 2011;2011. pii: 765029.

4. Aruoma OI. Free radicals, oxidative stress and antioxidants in human health and disease. J Am Oil Chem Soci 1998; 75:199-212.

5. Aruoma OI. Methodological consideration for characterizing potential antioxidant actions of bioactive components in food plants. Mut Res 2003; 523:9-20.

6. Zhu YZ, Huang SH, Tan BKH, Sun J, Whiteman M and Zhu YC. Antioxidants in Chinese medicines: A biochemical perspective. Nat Prod Rep 2004; 21:478-489.

7. Park Y, Nam S, Yi HJ, Hong HJ and Lee M. Dietary n-3 polyunsaturated fatty acids increase oxidative stress in rats with intracerebral hemorrhagic stroke. Nutr Res 2009; 29: 812-818.

8. Devasagayam TP, Boloor KK and Ramasarma T. Methods for estimating lipid peroxidation: an analysis of merits and demerits. Indian J Biochem Biophys 2003; 40(5): 300-308.

9. Repetto MG and Liesuy SF. Antioxidant properties of natural compounds used in popular medicine for gastric ulcers. Braz J Med Biol Res 2002; 35:523-534.

10. Sabu MC and Kuttan R. Antidiabetic activity of medicinal plants and its relationship with their antioxidant property. J Ethnopharmacol 2002; 81:155-160.

11. Tsao AS, Kim ES and Hong WK. Chemoprevention of cancer. Cancer J Clin 2004; 54:150-180.

12. Delanty $\mathrm{N}$ and Dichter Ma. Antioxidant therapy in neurologic diseases. Arch Neurol 2000; 57:1265-1270.

13. Deng GF, Lin X, Xu XR, Gao LL, Xie JF and Li HB. Antioxidant capacities and total phenolic contents of 56 vegetables. J Funct Food 2013; 5: 260-266. 
14. Guo YJ, Deng GF, Xu XR, Wu S, Li S, Xia EQ, et al. Antioxidant capacities, phenolic compounds and polysaccharide contents of 49 edible macro-fungi. Food Funct 2012; 3: 1195-1205.

15. Ameh SJ, Obodozie OO, Inyang US, Abubakar MS and Garba M. Current phytotherapy - A perspective on the science and regulation of herbal medicine. J Med Plants Res 2010; 4(2):072-081.

16. Oz AT and Kafkas E. Phytochemicals in fruits and vegetables. In: Super Food and Functional Food - An Overview of Their Processing and Utilization. In Tech; 2017; 175-184.

17. Brewer MS. Natural Antioxidants: Sources, Compounds, Mechanisms of Action, and Potential Applications. Comp Rev Food Sci and Food Safety. 2011;10: 221-247.

18. Reuland DJ, McCord JM and Hamilton KL. The role of Nrf2 in the attenuation of cardiovascular disease. Exerc Sport Sci Rev 2013; 41: 162-168.

19. Costa A, Garcia-Diaz DF, Jimenez P and Silva PI. Bioactive compounds and health benefits of exotic tropical red-black berries. J Funct Food 2013; 5: 539-549.

20. He DX, Ru XC, Wen L, Wen YC, Jiang HD, Bruce IC, et al. Total flavonoids of Flos Chrysanthemi protect arterial endothelial cells against oxidative stress. J Ethnopharmacol 2012; 139: 68-73.

21. Liqiang S, Li E, Wang F, Wang T, Qin Z, Niu S, et al. Quercetin increases macrophage cholesterol efflux to inhibit foam cell formation through activating PPARY-ABCA1 pathway. Int J Clin Exp Pathol 2015; 8(9): 10854-10860.

22. Kim HJ, Cha BY, Park IS, Lim JS, Woo JT and Kim JS. Dehydroglyasperin $\mathrm{C}$, a component of liquorice, attenuates proliferation and migration induced by platelet-derived growth factor in human arterial smooth muscle cells. Br. J Nutr 2013; 110: 391-400.

23. Thushara RM, Hemshekhar M, Santhosh MS, Jnaneshwari S, Nayaka SC, Naveen S, et al. Crocin, a dietary additive protects platelets from oxidative stress-induced apoptosis and inhibits platelet aggregation. Mol Cell Biochem 2013; 373: 73-83.

24. Chan J, Yuen A, Chan R and Chan SW. A review of the cardiovascular benefits and antioxidant propertiesnof allicin. Phytother Res 2013; 27: 637-646.

25. Barbosa A, Silveira GD, deMenezes I, Neto J, Bitencurt J, Estavam CD, etal. Antidiabetic effect of the Chrysobalanusicaco L. aqueous extract in rats. J Med Food 201; 16: 538-543.

26. Dembinska Kiec A, Mykkanen O, KiecWilk B and Mykkanen H. Antioxidant phytochemicals against type 2 diabetes. $\mathrm{Br} \mathrm{J}$ Nutr 2008; 991: S109-S117.

27. Tiwari AK, Jyothi AL, Tejeswini VB, Madhusudana K, Kumar DA, Zehra $A$, et al. Mitigation of starch and glucose-induced postprandial glycemic excursion in rats by antioxidant-rich greenleafy vegetables juice. Pharmacogn Mag 2013; 9S: 66-73.

28. Chuang CC and McIntosh MK. Potential mechanisms by which polyphenol-rich grapes prevent obesity-mediated inflammation and metabolic diseases. Annu Rev Nutr 2011; 31: 155-176.

29. Xie S, Sinha RA, Singh BK, Li GD, Han WP and Yen PM. Resveratrol induces insulin gene expression in mouse pancreatic alpha-cells. Cell Biosci 2013; 3: 47.

30. Kazemian M, Abad M, Haeri M, Ebrahimi M and Heidari R. Antidiabetic effect of Capparis spinosa $\mathrm{L}$. root extract in diabetic rats. Avicenna J Phytomed 2015; 5(4): 325-332.

31. Kooti W, Farokhipour M, Asadzadeh Z, Larky DA and AsadiSamani M: The role of medicinal plants in the treatment of diabetes: a systematic review. Electron Physician 2016; 8(1): 1832-1842.

32. Rasool M, Malik A, Qureshi MS, Manan A, Pushparaj PN, Asif M, et al. Recent updates in the treatment to neurodegenerative disorders using natural compounds. Evid Based Complement Altern Med 2014; 2014: Article ID 979730.

33. Kumar S, Maheshwari KK and Singh V. Effects of Mangifera indica, fruit extract on cognitive deficits in mice. J Environ Biol 2009; 30: 563-566.

34. Ansari MA, Abdul HM, Joshi G, Opii WO and Butterfield DA. Protective effect of quercetin in primary neurons against $A$ beta (1-42): Relevance to Alzheimer's disease. J Nutr Biochem 2009; 20: 269-275.

35. Davinelli S, Sapere N, Zella D, Bracale R, Intrieri M, Scapagnini G. Pleiotropic protective effects of phytochemicals in Alzheimer's disease. Oxid Med Cell Longev 2012;2012:386527.

36. Akram M and Nawaz A. Effects of medicinal plants on Alzheimer's disease and memory deficits. Neural Regen Res 2017; 12(4): 660-670.

37. Poulose SM, Miller MG and Shukitt-Hale B. Role of walnuts in maintaining brain health with age. J Nutr 2014; 144; 561S-566S.

38. Mishra $T$ and Bhatia A. Augmentation of expression of immunocytes functions by seed extract of Ziziphus mauritiana (Lamk.). J Ethanopharmaco 2010; 127:345-349.

39. Talero E, Avila-Roman J and Motilva V. Chemoprevention with phytonutrients and microalgae products in chronic inflammation and colon cancer. Curr Pharm Des 2012; 18: 3939-3965.

40. Andlujar I, Recio MC, Giner RM, Cienfuegos-Jovellanos E, Laghi S, Muguerza B and Rios JL. Inhibition of ulcerative colitis in mice after oral administration of a polyphenol-enriched cocoa extract is mediated by the inhibition of STAT1 and STAT3 phosphorylation in colon cells. J Agric Food Chem 2011; 59: 6474-6483.

41. Orsi PR, Bonamin F, Severi JA, Santos RC, Vilegas W, HirumaLima CA, et al. Hymenaea stigonocarpa Mart. ex Hayne: A Brazilian medicinal plant with gastric and duodenal anti-ulcer and anti-diarrheal effects in experimental rodent models. J Ethnopharmacol 2012; 143: 81-90.

42. Triantafyllidi A, Xanthos T, Papalois A and Triantafillidis JK. Herbal and plant therapy in patients with inflammatory bowel disease. Ann Gastroenterol 2015; 28(2): 210-220.

\footnotetext{
Authors Contribution:

TM- Idea and Manuscript Writing; AKK- Manuscript Editing; SDP- Helped in Data Collection; GSC- Helped in Data collection; SD- Helped in Data Collection; PKS-Helped in Data Collection

Work attributed to:

Aureus University

Orcid ID:

Dr. Ananda Kumar Kondepati - (D) https://orcid.org/0000-0001-8066-3768

Dr. Shalini Devaprasad Pasumarthi - https://orcid.org//0000-0003-4211-0366

Dr. Tulika Mishra - io https://orcid.org/0000-0001-6119-8792

Mr. Pratik Kumar Singh - https://orcid.org/0000-0001-6423-6352

Source of Support: Nil, Conflict of Interest: None declared.
} 